\title{
REVIEWS
}

$\mathrm{T}$ he maps of our everyday lives are much more than just linear lists of place names. Instead, their colours, symbols, contours and grid lines seek to describe different types of landscape, and to depict the spatial relationships between structural and functional landmarks of the environment (Fig. 1). It was the combination of photography and aviation that revolutionized mapmaking in the early part of this century. In much the same way, it is fluorescence microscopy and digital imaging (Box 1) in combination with molecular genetics that is driving our emerging view of the genome in space and time.

Condensed for mitosis but allowing a little latitude

Chromosomes trapped in mitosis are our most familiar visual representation of the genome. Their banded appearance, when stained in the appropriate way, can be used to give us clues about the different types of chromatin environment within the genome (from C-, G- and R-bands, through to T-bands) and about global patterns of DNA organization ${ }^{1,2}$. The concentration of vertebrate genes into R- and T-bands that replicate early in S phase, that are GC-rich and that are packaged with hyperacetylated nucleosomes ${ }^{3-7}$ suggests a functional and structural segregation of the genome into 5-10 Mb-sized chunks.

The condensed and rod-like appearance of metaphase chromosomes have made them ideal reference points for mapping DNA sequences relative to the longitudinal chromosome axis by fluorescence in situ hybridization (FISH). There have also been reports that specific loci occupy fixed lateral positions on each sister chromatid, that is, either internal, median or external, relative to the long axis ${ }^{8}$. Such mirror symmetry supports the idea that sister chromatids are helices of opposite handedness - a conclusion also reached by observing the folding path of the chromosome scaffold at the axial core of each chromatid?. Electron micrographs of a halo of DNA loops surrounding this metaphase scaffold, in chromosomes that have been experimentally decondensed by extraction of soluble proteins, are compelling visual evidence favouring a radial loop/scaffold model of metaphase chromosome structure ${ }^{10}$. Major proteins of this scaffold are toposiomerase II $\alpha$ and ScII. The former is required for decatenation of sister chromatids and for chromosome condensation; the latter is an SMC protein, some of whose family members are components of complexes needed for the condensation of mitotic chromosomes and for the maintenance of sister-chromatid cohesion ${ }^{11}$. The chromosome core can also be seen, although in less exquisite detail, by light microscopy. Combined with FISH, this has enabled topographical descriptions of the human genome to be made within metaphase chromosomes ${ }^{12}$. Specific DNA segments are consistently associated with the axial core on both chromatids, with loops of DNA extending out for $\sim 200 \mathrm{~kb}$ either side (Fig. 2). The sites of association between DNA and this chromosome core correlate with the position and frequency of origins of replication in the genome ${ }^{12,13}$. Could the folding of the genome within metaphase chromosomes be directing the position of potential origins in the next cell cycle, or is it just reflecting the position of origins in the

\section{Putting the genome on the} map

\author{
JOANNA M. BRIDGER (joannab@hgu.mrc.ac.uk) \\ WENDY A. BICKMORE (wendy@hgu.mrc.ac.uk)
}

The first complete genomic sequence of a eukaryote (Saccharomyces cerevisiae) has already been accomplished. It is estimated that the sequence of the buman genome will be known early in the next millennium. Yet it is already apparent that, despite their immense length, these linear primary sequence maps will be inadequate descriptions of the eukaryotic genome, be it of a budding yeast or a buman. To reflect our growing awareness of the importance of spatial context in chromosome function and in gene expression we argue that a more complete map of the genome should seek to embody the richness of information that we expect of the maps we use to navigate our way around the outside world.

preceding one? In either case, the mirror symmetry of higher-order sister chromatid folding prompts us to think about how this could be established and propagated through DNA replication. The topology is either established before or during replication, or it is set up anew and symmetrically on the sister chromatids after DNA replication and before mitosis. Both of these options challenge our knowledge about how chromatin structure is maintained through cell divisions. While the mechanism for making an accurate copy of DNA is inherent within the structure of the double helix itself, such an elegant solution does not suggest itself for chromosome structure.

Because parts of the genome are buried within the interior of the mitotic chromosome, by default, other sequences must be exposed on the surface and available to interact with specific proteins found there. A subset of nuclear proteins, many of unknown function but with DNA-binding properties or containing DNAbinding motifs, coat mitotic chromosomes and might interact with exposed sequences ${ }^{14}$. It is not clear whether these proteins are just hitching a ride on the chromosomes into the interphase nucleus or whether they have a more specific role in protecting the genomic DNA from cellular components during open mitosis, or in directing genome organization in the subsequent interphase. At the end of mitosis, the new nuclear envelope is built upon a foundation of chromosomes and integral membrane components of the nuclear envelope, such as lamina-associated proteins (LAPs) and the lamin B receptor (LBR), are recruited to the chromosome surface ${ }^{15,16}$. Their role in establishing the organization of the interphase nucleus will be discussed below.

\section{Staking out territory}

Once in interphase, chromosomes are generally considered to be less condensed than their mitotic counterparts, but individual chromosomes are still discrete entities and not significantly intermingled with each other ${ }^{17-19}$, hence the term chromosome territory has 


\section{REVIEWS}

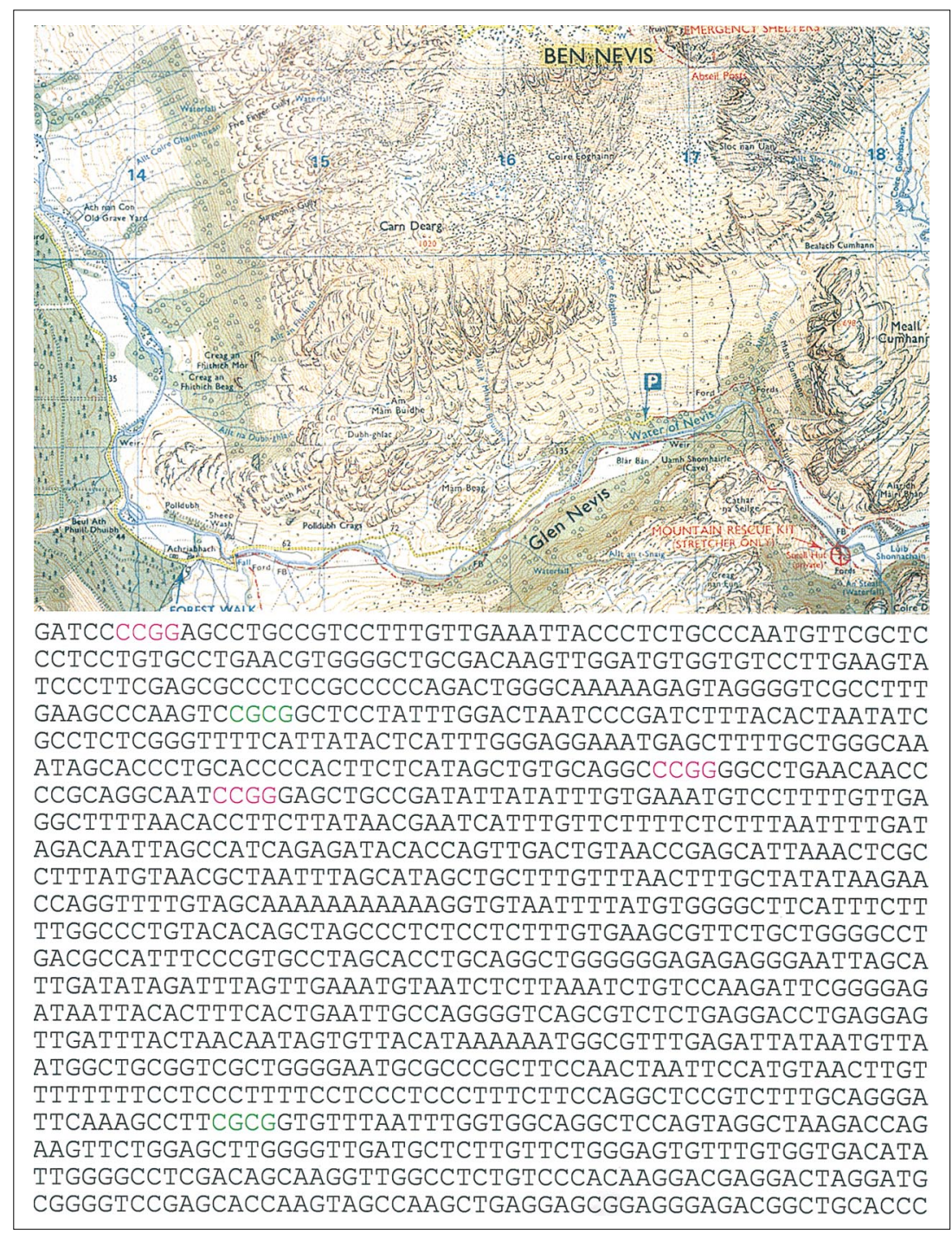

FIGURE 1. Which type of map? At the top is a map of Glen Nevis and the southern slopes of Ben Nevis (Scotland's highest mountain) that shows regions of the local environment that are river, bog, forest or rock. The relationships between place names as well as structural and functional features of the landscape are represented in this map. The lowest and highest points of the terrain are depicted using contour lines. (Reproduced from the Ordnance Survey material with the permission of The Controller of Her Majesty's

Stationery Office, (C) Crown Copyright Licence No. MC 88276M.) Below is a portion of the

human genome map (from a $200 \mathrm{~kb}$ stretch of sequence from human chromosome

11p13). The complete human genome map will be 2.5 million times longer than the piece shown here. The nucleotide sequence does not give us any clues about how this DNA may be packaged up into chromatin or chromosomes inside of cells, nor to which other parts of the genome or nuclear compartment it may be in physical proximity.
(C. Muenkel and J. Langowski, pers. commun.). We still do not have a feel for how much of a chromosome might actually lie outside of what we visually define as the territory, delineated using complex chromosome-painting probes.

In the same way that mitotic chromosomes have an inner core and outer surface to which discrete parts of the genome can be spatially mapped, so the volume of an interphase chromosome territory might be considered to have an interior and a periphery. Do specific parts of the genome map to these sites, and how much remodelling and refolding of the territories occurs during the cell cycle and during differentiation? Threedimensional interphase genome mapping has been initiated and, although the number of individual sequences analysed thus far is small, patterns are emerging. Of three coding regions of the human genome examined, all were located at the surface of their respective chromosome territories, independently of transcriptional status ${ }^{24}$. The most extreme interpretation of these data is that most, or all, genes lie on the surface of chromosome territories (Fig. 4a), begging the question of what sequences comprise the interior volume. There is plenty of non-coding DNA in much of the mammalian genome to fill this space (half the volume of a sphere is located in the shell comprising only the outer one-fifth of its radius). However, this model has more profound topographical implications in regions of the mammalian genome (e.g. the MHC on human chromosome 6p), where genes are packed very closely over many $\mathrm{Mb}$, in other vertebrate genomes where gene density is high (e.g. Fugu and the microchromosomes of birds)

been widely adopted (Fig. 3). Moreover, sub-chromosomal domains (for example, individual $\mathrm{p}$ and $\mathrm{q}$ chromosome arms, chromosome bands, and even smaller domains) are maintained within their own sub-territories at interphase ${ }^{20-22}$ (D. Zink, pers. commun.), suggesting that the genome segmentation revealed through metaphase chromosome bands is translated into interphase nuclear space. Local compartmentalization of chromatin is consistent both with a multi-loop model ${ }^{20}$ of interphase chromosome structure (akin to the radialloop model of the metaphase chromosome) and with the random-walk or giant-loop model ${ }^{23}$, although these two models make different predictions about the extent of compartmentalization at different levels of resolution and in eukaryotes with little non-coding DNA (e.g. budding yeast). Are the chromosome territories in these latter cases all surface and no interior? It is clear that more work needs to be done to test these ideas, by studying the spatial distribution of more loci and larger, morecontiguous genomic regions, and also by testing the distribution of other global genomic features, such as CpG islands, which coincide with concentrations of genes.

What is appealing about having genes at the surface of chromosome territories is that it lessens the problem of how proteins that process RNA can find genes amongst large tracts of non-coding DNA. The model might also be extended to include distant regulatory elements and factors that bind them. It was proposed 


\section{REVIEWS}

that transcription, RNA processing and transport occur in a space between territories called the interchromosomal domain (ICD) compartment, which connects with the cytoplasm through channels to the nuclear pores $17,25,26$ (Figs 3 and 4 a). If this is extended to include activities that act on all DNA, such as replication and repair, then it would demand extensive remodelling of the chromosome territory during the cell cycle, so that all sequences could spend some time on the chromosome surface. The localization of sites of DNA replication within the interior of territories ${ }^{19,21}$ indicates that replication is not confined to the territory surface and, so, does not demand that there be massive movement of DNA at S-phase towards enzyme complexes on the chromosome surface.

Predictions of the ICD model are that nascent RNAs, RNA polymerases and splicing complexes should be found at the frontier of chromosome territories and not in their interior. Accumulations of specific RNAs have been seen at the border of the territory of the chromosome from which they originated, next to their cognate gene loci 25,27 . Most of these studies have examined transcripts originating from integrated copies of viral genomes. Although transcripts from the $1 \alpha 1$ collagen gene on human chromosome17 have been examined in fibroblasts where the gene is very actively transcribed ${ }^{27}$, it still remains to be seen whether the same is generally true of endogenous mammalian genes and their transcripts. Components of the splicing machinery in transcriptionally active cells are also concentrated outside of the chromosome territories 25 .

Arguing against the simple ICD model, poly(A) RNA is not found exclusively outside chromatin domains, although it appears to concentrate in parts of the nucleus with low DNA density ${ }^{28}$, and ongoing transcription can be seen to occur deep within chromosome territories (P. Verschure and R. van Driel, pers. commun.). If genes concentrate on the chromosome surface then a prediction is that domains of early and late replication (gene-rich and gene-poor) will partition to the periphery and interior of territories, respectively. However, early- and late-replicating domains are distributed throughout chromosome territories, implying that early-replicating (and so potentially gene-rich) DNA can be found within the chromosome interior ${ }^{21}$ (D. Zink, pers. commun.). The only place where earlyreplicating DNA was found preferentially at the surface of a chromosome territory was on the inactive $\mathrm{X}\left(\mathrm{X}_{\mathrm{i}}\right)^{21}$.

To accommodate these discrepancies, and to explain the three-dimensional properties of $\mathrm{X}_{\mathrm{i}}$ and the active $\mathrm{X}$ $\left(\mathrm{X}_{\mathrm{a}}\right)$, the ICD model has been modified to broaden the concept of what qualifies as the surface of an interphase chromosome. Simple observations of $\mathrm{X}_{i}$ in flattened nuclei were always taken as evidence that this silenced chromosome was more condensed than its active counterpart. However, surprisingly, in three-dimensionally preserved nuclei $X_{i}$ and $X_{a}$ are calculated to take up the same nuclear volume as each other. But $\mathrm{X}_{\mathrm{i}}$ has a rounder and more smooth morphology, whereas $X_{a}$ has a more open structure with furrows that run from the territory surface to the inside of the chromosome ${ }^{29}$. These differences could either relate directly to the contrasting transcriptional activities of $\mathrm{X}_{\mathrm{a}}$ and $\mathrm{X}_{\mathrm{i}}$ or to the coating of XIST RNA on the inactive X (Ref. 27). If the

\section{Box 1. Digital imaging}

Digital imaging has transformed the field of cellular genetics virtually replacing photomicroscopy. Light (photons) emitted by fluorescence samples is detected and stored as digital data; then, a computer is required to transform the raw data into viewable and manipulatable images. Studies can be performed in two, three and four dimensions, and there are various fluorescence-detecting systems that can be employed, such as confocal laser scanning microscopy (CLSM), multiphoton excitation microscopy (MEM) and video microscopy (VIM). For more details, see Ref. 74 .

\section{CLSM}

The principle of CLSM is that an image of a particular section is collected without the out-of-focus fluorescence being included. This is achieved primarily by passing the emitted fluorescence generated by laser excitation through a small aperture. Confocal microscopy has been widely used for three-dimensional analyses, collecting stacks of twodimensional confocal optical sections.

\section{MEM}

As with CLSM, lasers are used as the source of excitation of the fluorophore. Short pulses of long-wavelength radiation are used. No out-of-focus fluorescence is generated because there is only enough energy to excite the fluorophore where the beam is focused.

\section{VIM}

Video microscopy has become very popular and most researchers use cooled, charge-coupled device (CCD) cameras, enabling the detection of weak signals and minimal exposure of the sample to the excitation source, making it a method of choice when performing real-time analyses. Three-dimensional analyses are also possible by collecting a series of sections through the sample. These images will include the out-of-focus fluorescence, but this can be removed by deconvolution algorithms. The computation required for this removal is now possible using commercially available, user-friendly software packages.

ICD compartment is extended to include such inlets into the body of chromosomes, it is still possible that transcription apparently occurring within chromosome territories is actually on the chromosome 'surface' (Fig. 4a). Indeed, transcripts of $1 \alpha 1$ collagen apparently within the chromosome 17 territory often coincide within holes in the chromosome paint ${ }^{27}$.

\section{Global positioning}

Condensed heterochromatin can be seen within the nuclei of many eukaryotes, and even within euchromatin there are differences in interphase chromatin condensation between, for example, G- and R-bands ${ }^{30}$. The more-condensed regions of the nucleus probably equate with compartments that are repressive for transcription, and so the position of a gene within the nucleus might influence its activity. In turn, the position that genes can adopt within the nuclear space might be dictated by, or constrained by, sequences that they are joined to in cis (i.e. their position along a chromosome $)^{31-33}$. Therefore, it is necessary for us to move from genome maps in which each DNA molecule (chromosome) is portrayed in isolation, to maps in which the spatial relationships between sequences on the same and on different chromosomes, and between sequences and the nuclear space, can be depicted. 


\section{REVIEWS}

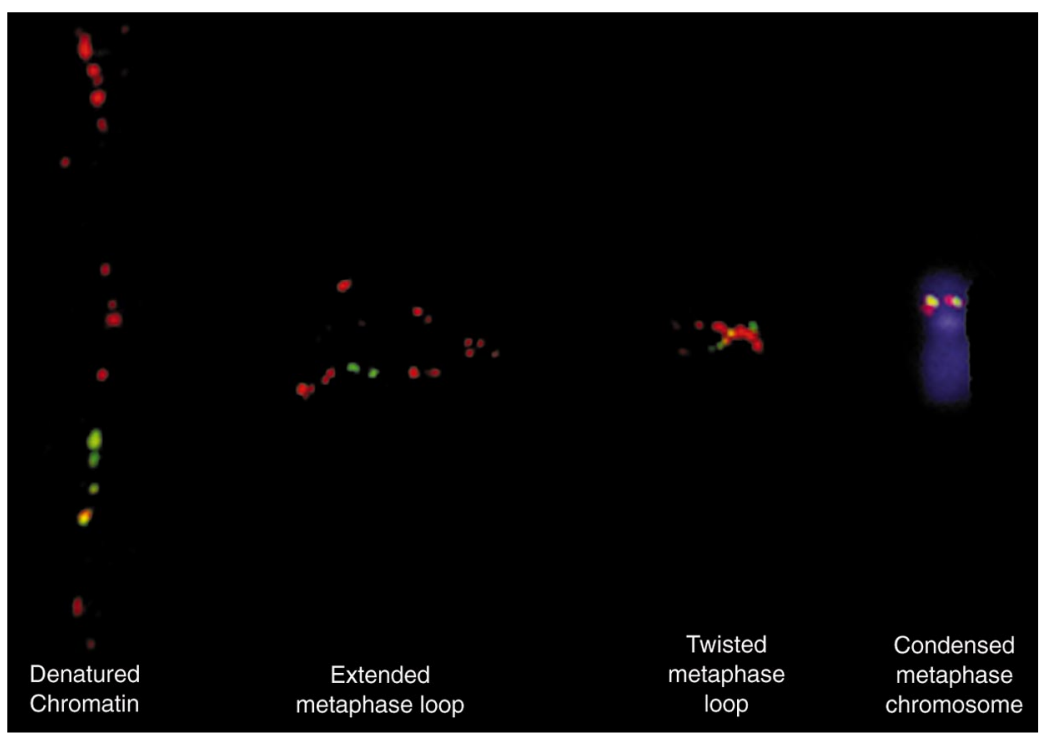

FIGURE 2. Unravelling genome topography. FISH signals from a $450 \mathrm{~kb}$ YAC (red) and an overlapping cosmid (green) that originate from human chromosome 11p13. The nucleotide sequence in Fig. 1 is contained in the middle of the YAC and the cosmid is $\sim 75 \mathrm{~kb}$ proximal of it. The template for hybridization at the extreme right was a fully condensed metaphase chromosome. Both probes colocalize at the same point along

the longitudinal chromosome axis and there is no lateral spatial resolution. The

$450 \mathrm{~kb}$ of DNA detected by the YAC is represented by a single spot of hybridization on each chromatid. At the other extreme, on the far left the probes have been cohybridized to denatured and decondensed chromatin fibres so that the position of the cosmid along the length of the YAC can now be determined; all of the information is still linear as in Fig. 1. In between, the probes have been hybridized to chromatids from metaphase chromosomes that have had soluble proteins extracted from them to differing degrees. The YAC signal now has a distinctive ' $\mathrm{V}$ ' shape (second from left) with the path of the DNA bent through an acute angle at the chromosome core ${ }^{12}$. The sequence in Fig. 1 maps close to this point, the cosmid maps mid way along a chromosome loop. To the right of this a similar loop is seen, but with some twisting or supercoiling retained in the loop.

In position effect variegation (PEV), genes become silenced by their stochastic interaction with heterochromatin. In classical PEV this is a cis effect in which sequences (often tandem repeats) that can nucleate complexes of heterochromatin proteins are juxtaposed next to endogenous gene loci ${ }^{33}$, or in which transgenes are integrated close to endogenous blocks of satellite DNA (Refs 34-36). In special situations, silencing can occur in trans, that is, there does not have to be satellite or repetitive DNA covalently joined to a gene. In Drosophila, homologous chromosome pairing ensures that the juxtaposition in cis of heterochromatin close to one allele of the brown gene $\left(B w^{D}\right)$ is enough to move the other (wild-type) allele into physical association with centric heterochromatin 31,32 and so bring about its repression.

Are there similar areas of repression within the mammalian nucleus? Many transgenes in the mouse have been found to insert close to pericentric heterochromatin, and they are frequently subject to PEV at these sites ${ }^{34-36}$. The physical proximity to heterochromatin on the chromosomal DNA molecule is mirrored in the interphase nucleus (Fig. 4) ${ }^{35}$. Endogenous mouse genes, not close to heterochromatin in cis, can also locate to regions of the nucleus occupied by pericentric heterochromatin ${ }^{37}$. Transcriptionally inactive genes that encode various B-cell surface determinants and that map on different chromosomes colocalize together with the transcriptional regulator Ikaros in the nuclei of mouse B lymphocytes at sites of pericentric heterochromatin (Fig. 4b). At stages of B-cell differentiation, when the same genes are active, there is no colocalization ${ }^{37}$. Unanswered questions are: whether the colocalization is a cause or consequence of inactivation; whether (as is the case in $B w^{D}$ ) the silenced genes colocalize with heterochromatin only from their own chromosome; and, if not, how far away from the rest of their chromosome territory do these genes move when they take up residence in domains occupied by pericentric heterochromatin, and how much surrounding DNA and genes do they take with them (Fig. 4)?

Condensations of DNA visible with simple DNA stains are unlikely to be the only domains of silencing in mammalian nuclei. Proteins encoded by vertebrate homologues of the genes encoding the Polycomb group (PcG) proteins (e.g. BMI and HPH1/MPH1, M33, RING1) and interacting proteins, can colocalize in foci distinct from pericentric heterochromatin ${ }^{38}$, although some human PCG proteins associate with pericentric heterochromatin in some cell types (P. Freemont, pers. commun.). It will not be surprising if genes regulated by these complexes (e.g. Hox genes) map in space to these same nuclear domains. There seems to be no reason to suppose that gene silencing should have the monopoly on nuclear domains - there will probably be places in the nucleus where genes subject to common means of activation are brought together. The number of nuclear domains and bodies identifiable through accumulations of specific proteins is expanding ${ }^{39-41}$. We cannot assign functional significance to most of these domains yet, but some of them contain transcriptional activators and we should continue to watch these nuclear spaces as they converge with specific parts of the genomic sequence ${ }^{41}$.

\section{Close to the edge}

Just as genomic sequence can be mapped relative to the inner or outer compartments of individual chromosomes at mitosis and interphase, so sequence can also be mapped relative to obvious structural landmarks of the nucleus. The human rDNA-containing acrocentric chromosomes are intrinsic components of nucleoli, and other chromosomes that contain no rDNA also seem to localize within or close to this compartment (Fig. 3) ${ }^{42,43}$.

Towards the edge of the nucleus there is a concentration of heterochromatin and repressed genes ${ }^{37,44}$, and a dearth of poly (A) RNA and accumulations of splicing factors ${ }^{28}$. Direct genetic links between location at the nuclear periphery and repression of transcription are revealed by the roles of Sir3p and Sir $4 p$ in the 


\section{REVIEWS}

budding yeast 33 and by silencing that can be facilitated by anchoring a locus to the nuclear periphery in this organism 75 . At a more global level, regions of the mammalian genome replicating in mid- to late $S$ phase also concentrate towards the edge of the nucleus ${ }^{45-47}$. This has led to the suggestion that all mammalian chromosomes make some contact with the nuclear envelope ${ }^{48}$. This would mean that small, gene-rich and early-replicating chromosomes that also harbour rDNA (e.g. human chromosome 22 and one of the chicken microchromosomes 3,6 ), are strung out between the nucleolus and the nuclear periphery.

It is tempting to speculate that the decoration of mitotic chromosomes with integral nuclear membrane proteins (IMPs) leads to the positioning and possibly anchoring of heterochromatin or gene-poor domains at the nuclear envelope (Fig. 3). Many IMPs, such as LAP2 and LBR, have chromatin- and/or DNA-binding abilities. LBR tantalizingly adorns the surface of Chinese hamster mitotic chromosomes in a G-banding pattern ${ }^{16}$, and has an affinity for the heterochromatic protein HP1 (Ref. 49). Nuclear lamins themselves can also associate with mitotic chromosomes and bind to chromatin ${ }^{50}$.

The protein-DNA interactions involved in partitioning parts of the genome to either the nuclear periphery or interior and nucleolus clearly have major consequences on overall genome topology and function. The ultimate position of any chromosome or gene locus in the nucleus will be a trade-off of interactions between the genome and several different components of nuclear architecture. Changes in the localization of proteins and gene loci at the nuclear periphery, nucleolus or within the nucleus might be associated with dramatic changes in phenotype, such as cellular ageing and tumourigenesis ${ }^{51-54}$

\section{The bare bones of a map}

Components of the nuclear lamina and the nucleolus remain intact after nuclei have been extracted with a variety of agents55,56. As well as these durable constituents, it is hypothesized that there is a more extensive ultrastructure that withstands such treatments and that ramifies throughout the nucleus. Depending on methodology and ideology, such structures have been burdened with graphic labels such as nuclear matrix, scaffold or skeleton. The numerous arguments that have erupted from such studies generally centre around whether such sub-structure is a pre-formed framework within the nucleus with an organizational role, or whether it is merely a consequence of function and accumulations of protein complexes.

DNA sequences fractionating with the nuclear matrix (matrix attachment regions, MARs), scaffold (scaffold attachment regions, SARs) or skeleton (no collective acronym!) can be isolated biochemically. Gross differences in the characteristics of each of these groups of sequences are exposed by their distinctly different distributions in the genome ${ }^{57}$. The discord may arise through our tendency to assume equivalence between the scaffold, matrix and skeleton and their associated DNA sequences, whereas they each are probably very different in composition and function. The intrinsic mitotic chromosome scaffold might persist in a modified interphase form within chromosome territories. By

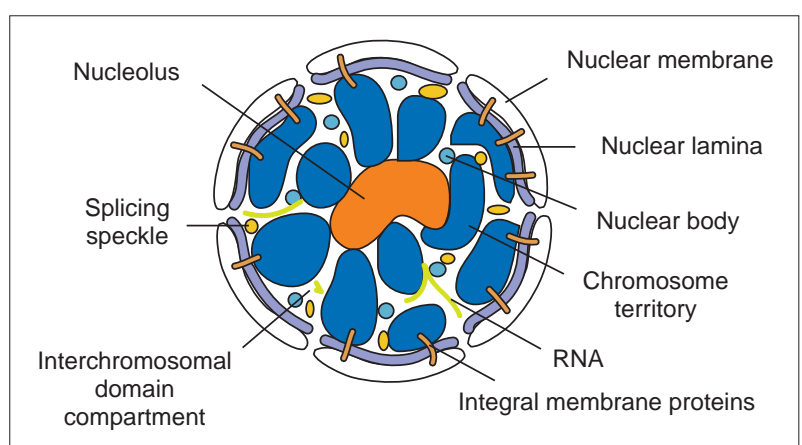

FIGURE 3. Model of interphase nuclear organization. The nucleus is comprised of a number of structural entities, compartments, concentrations of components involved in metabolic processes and, of course, chromosomes. The nuclear membrane contains integral membrane proteins such as lamin B receptor (LBR) and lamina associated protein 2 (LAP2) that might have a role in positioning chromosomes within the nucleus ${ }^{15,16,49}$. The nuclear lamina is subjacent to the inner nuclear envelope. Chromosomes are in territories (dark blue) and there appears to be little intermingling of chromosomes ${ }^{17-20}$. Between the chromosomes is the interchromosomal domain (ICD) compartment ${ }^{17,25,26}$, which contains various nuclear bodies, such as coiled bodies and PML bodies, specific species of RNA and components of the splicing machinery 25,26

contrast, many classical nuclear matrix components have no mitotic counterparts and are distributed within the ICD compartment58 and include RNA, and complexes that act on RNA (Refs 59, 60). Specific species of RNAs and actively transcribed genes also resist extraction in nuclear matrix preparations ${ }^{27,56}$.

It has been suggested that transcription occurs in immobilized factories that make up part of the nuclear skeleton. Each of these factories contains multiple active polymerases and, in principle, they could bring together templates derived from gene loci on different chromosomes ${ }^{61}$. Similarly, replication is also proposed to occur in factories of immobilized DNA polymerases ${ }^{62}$ at which replication is initiated from multiple origins simultaneously. Again, each factory could bring together replicons located on different chromosomes, but the coherence of pulse-labelled sites of replication through into metaphase and subsequent cell cycles suggests that these clustered replicons derive from the same chromosome and, indeed, the same subchromosomal region (band) ${ }^{19,22}$ (D. Zink, pers. commun.). Clusters of early-replicating (gene-rich) DNA resist extraction from the nucleus with high $s^{2}{ }^{22}$, and some origins of replication specifically co-map with sites of attachment to the nuclear matrix ${ }^{63}$.

\section{Maps on the move}

Every facet of genome organization we have discussed could be dynamic. The location of chromosomes, sub-chromosomal domains and specific loci inside the nucleus, and their associations with specific proteins, might be modified during the cell cycle and during differentiation. The concentration of Ikaros protein into foci in B cells is lost during $\mathrm{S}$ phase and, moreover, as immature B-lymphocyte cells progress to maturity the complement of genes associated with these foci changes ${ }^{37}$. Movements of centromeres and telomeres 


\section{REVIEWS}

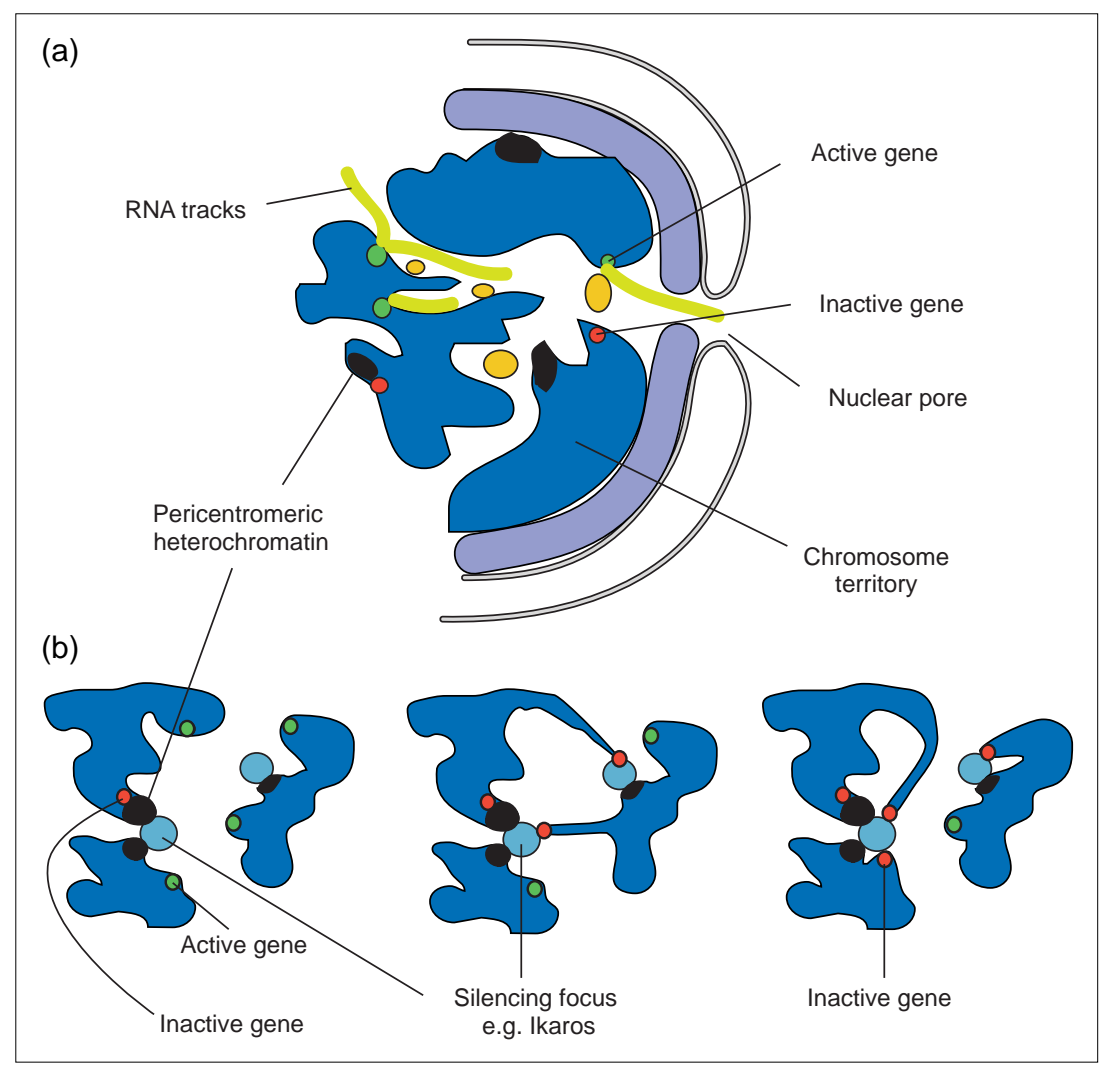

FigURE 4. Spatial localization of active and silenced genes in nuclei. (a) Active genes (green) have been seen on the surface of chromosome territories ${ }^{24}$. The ICD compartment is suggested to be where transcription and RNA processing occur and to be the channels through which RNA travels to be exported through nuclear pores $17,25,26$. Whether transcription takes place throughout chromosome territories or is only at the territory exterior is now being debated, but it is probable that chromosome territories have ICD channels extending into them ${ }^{27,29}$. (b) In the left panel, some genes are silenced (red) through their location, in cis, close to heterochromatin (black) ${ }^{33-36}$. In the middle and right panels, other silenced genes (red), although not located close to heterochromatin in cis, can nonetheless locate to parts of the nucleus occupied by heterochromatin. Concentrations of proteins (e.g. Ikaros) involved in the transcriptional regulation of such genes colocalize with the silenced genes ${ }^{37}$. We do not know whether such genes solely reside close to heterochromatin from their own chromosome territory (right panel) 31,32 or whether they can colocalize with heterochromatin from another chromosome territory (middle panel)

during the cell cycle have been recorded 64,65 . Pairing of homologous chromosomes in Drosophila does not occur in early embryonic stages ${ }^{32}$, and a fleeting association of homologous chromosomes, but only at imprinted loci, has been seen in late $S$ phase of mammalian cells ${ }^{66}$. Conversely, extrapolation from the regular arrangement of human chromosomes on the metaphase plate suggests a more static overall view of interphase chromosome arrangement ${ }^{67}$.

It is possible, but cumbersome, to take snapshots of the genome in cells fixed at different points during the cell cycle or during development. It is more elegant, and more powerful, to visualize specific genomic regions of living cells in real-time. Whole chromosome territories have been revealed by microinjecting fluorescently tagged nucleotides into cells, allowing these cells to complete several division cycles, and so to segregate (through semi-conservative replication) the label to one or a few chromosomes ${ }^{19}$. The problem with this approach is that it is impossible to tell which chromosome is which inside the living cell in this way.
A single transcriptionally active locus has been visualized in living Drosophila embryos by the microinjection of fluorescently labelled antibody directed against a protein recruited to the locus after heatshock. Considerable and distinct types of movements of this locus were recorded 68 .

New avenues of investigation are coming from the use of green fluorescent protein (GFP). Specific chromosomal proteins have been fused to GFP so that the sites of these proteins, and we assume the normal DNA sequences that they associate with, can be visualized in living cells. For example, in human cells expressing the $\alpha$-satellite binding protein CENP-B fused with GFP (Ref. 69), the elasticity of individual centromeric domains contrasts with the static relationship of centromeres with each other. Similarly, GFP-topoisomerase II in Drosophila has been used to address the question of how free or constrained chromosomes are to diffuse within the nucleus ${ }^{70}$. The movements recorded appear to be passive (compatible with Brownian motion) rather than actively directed by cellular activities. The important findings of these experiments are that the diffusion constants measured, although several orders of magnitude slower than DNA in solution, are sufficiently large to allow significant movements during the course of interphase, but they are also low enough that chromatin can only diffuse slowly within a limited subregion of the nucleus. This combination of constraint and diffusion leads the authors of this study to the conclusion that nuclear architecture is an important factor in determining whether any two loci are likely to meet. It also means that specific localization of DNA segments might be most effectively established as cells exit mitosis and before the fully formed interphase nucleus constrains large-scale movements.

These conclusions were confirmed in budding yeast by targeting GFP to novel binding sites engineered into the yeast genome ${ }^{70}$ and a similar approach has recently been extended into mammalian cells. However, one caveat of these studies is that detection of GFP binding in the mammalian genome necessitates large amplified arrays of binding sites. The aberrant packaging of such sequences in the mammalian nucleus is manifest by their appearance in the nucleus as homogeneously staining regions ${ }^{71,72}$, leaving open the possibility that their recorded dynamic movements and condensation are not representative of normal chromatin. 


\section{REVIEWS}

Future aims must be to vivify normal endogenous loci, either by improving the sensitivity of the existing GFP-based systems or by using other directed reporters that are compatible with living cells ${ }^{73}$. It is clear that unfolding genome topography will continue to drive our understanding of mechanisms of gene regulation, genome instabilities and rearrangements. It might also allow us to manipulate the genome in more sophisticated and subtle ways. Ptolemy urged the map-makers of the second century AD to 'survey the whole in its just proportions' and to represent in pictures the whole known world together with the phenomena contained therein'. There can be no better call to arms for genome mappers of the third millennium.

\section{Acknowledgements}

We thank P. Cook, R. van Driel, P. Freemont, C. Muenkel, A. Visser and D. Zink for access to their unpublished data, and R. Allshire, V. van Heyningen and N. Hastie for their comments. Lastly, we thank the Ordnance Survey for permission to reproduce part of the OS map of Glen Nevis and for producing maps to aspire to!

\section{References}

1 Craig, J.M. and Bickmore, W.A. (1993) BioEssays 15, 349-354

2 Saitoh, Y. and Laemmli, U.K. (1994) Cell 76, 609-622

3 Craig, J.M. and Bickmore, W.A. (1994) Nat. Genet. 7, 376-382

4 Saccone, S., De Sario, A., Della Valle, G. and Bernardi, G. (1992) Proc. Natl. Acad. Sci. U. S. A. 89, 4913-4917

5 Jeppesen, P. and Turner, B. (1993) Cell 74, 281-289

6 McQueen, H.A. et al. (1996) Nat. Genet. 12, 321-324

7 Cross, S. et al. (1997) Genomics 40, 454-461

8 Baumgartner, M. et al. (1991) Cell 64, 761-766

9 Boy de la Tour, E. and Laemmli, U.K. (1988) Cell 55, 937-944

10 Paulson, J.R. and Laemmli, U.K. (1977) Cell 12, 817-828

11 Heck, M.M. (1997) Cell91, 5-8

12 Bickmore, W.A. and Oghene, K. (1996) Cell 84, 95-104

13 Lawlis, S.J. et al. (1996) J. Cell Biol. 135, 1-12

14 Hernandez-Verdun, D. and Gautier, T. (1994) BioEssays 16, 179-185

15 Foisner, R. and Gerace, L. (1993) Cell 73, 1267-1279

16 Pyrpasopoulou, A. et al. (1996) EMBO J. 15, 7108-7119

17 Cremer, T. et al. (1993) Cold Spring Harbor Symp. Quant. Biol. 58, 777-792

18 Leitch, A.R. et al. (1990) J. Cell Sci. 95, 335-341

19 Zink, D. et al. (1998) Hum. Genet. 102, 241-251

20 Dietzel, S. et al. (1998) Chromosome Res. 6, 25-33

21 Visser, A. et al. Exp. Cell Res. (in press)

22 Jackson, D. and Pombo, A. (1998) J. Cell Biol. 140, 1285-1295

23 Sachs, R.K. et al. (1995) Proc. Natl. Acad. Sci. U. S. A. 92 2710-2714

24 Kurz, A. et al. (1996) J. Cell Biol. 135, 1195-1202

25 Zirbel, R.M. et al. (1993) Chromosome Res. 1, 93-106

26 Bridger, J.M., Herrmann, H., Muenkel, C. and Lichter, P. (1998) J. Cell Sci. 111, 1241-1253

27 Clemson, C.M., McNeil, J.A., Willard, H.F. and Lawrence, J.B. (1996) J. Cell Biol. 132, 259-275

28 Carter, K.C., Taneja, K.I. and Lawrence, J.B. (1991) J. Cell Biol. 115, 1191-1202

29 Eils, R. et al. (1996) J. Cell Biol. 135, 1427-1440

30 Yokota, H., Singer, M.J., van den Engh, G.J. and Trask, B.J. (1997) Chromosome Res. 5, 157-166

31 Csink, A.K. and Henikoff, S. (1996) Nature 381, 529-531

32 Dernburg, A. et al. (1996) Cell 85, 745-759

33 Maillet, L. et al. (1996) Genes Dev. 10, 1796-1811

34 Milot, E. et al. (1996) Cell 87, 105-114
35 Dobie, K.W. et al. (1996) Proc. Natl. Acad. Sci. U. S. A. 93, 6659-6664

36 Festenstein, R. et al. (1996) Science 271, 1123-1125

37 Brown, K.E. et al. (1997) Cell 91, 845-854

38 Satijn, D.P. et al. (1997) Mol. Cell. Biol. 17, 4105-4113

39 Boddy, M.N., Duprez, E., Borden, K.L. and Freemont, P.S (1997) J. Cell Sci. 110, 2197-2205

40 Brett, D. et al. (1997) Hum. Mol. Genet. 6, 1559-1564

41 Pombo, A. et al. (1998) EMBO J. 17, 1768-1778

42 Weitspolthammer, K. et al. (1996) Cytogenet. Cell Genet. 73, 108-113

43 Bridger, J.M., Kill, I.R. and Lichter, P. (1998) Chromosome Res. 6, 1133-1142

44 Belmont, A., Bignone, F. and Ts'o, P.O.P. (1986) Exp. Cell Res. $165,165-179$

45 Fox, M.H. et al. (1991) J. Cell Sci. 99, 247-253

46 Kill, I.R. et al. (1991) J. Cell Sci. 100, 869-876

47 O'Keefe, R., Henderson, S.C. and Spector, D.L. (1992) J. Cell Biol. 116, 1095-1110

48 Ferreira, J., Paolella, G., Ramos, C. and Lamond, A. (1997) J. Cell Biol. 139, 1597-1610

49 Ye, Q. et al. (1997) J. Biol. Chem. 272, 14983-14989

50 Taniura, H., Glass, C. and Gerace, L. (1995) J. Cell Biol. 131, 33-44

51 Imai, S.I. et al. (1997) Mol. Biol. Cell 8, 2407-2419

52 Gotta, M. et al. (1997) EMBO J. 16, 3243-3255

53 Linares-Cruz, G. et al. (1998) Proc. Natl. Acad. Sci. U. S. A. 95, 1131-1135

54 Krystosek, A. (1998) Exp. Cell Res. 241, 202-209

55 Capco, D.G., Wan, K.M. and Penman, S. (1982) Cell 29, $847-858$

56 Gerdes, M.G., Carter, K., Moens, P.T. and Lawrence, J.B. (1994) J. Cell Biol. 126, 289-304

57 Craig, J.M., Boyle, S., Perry, P. and Bickmore, W.A. (1997) J. Cell Sci. 110, 2673-2682

58 Razin, S.V. and Gromova, I.I. (1995) BioEssays 17, 443-450

59 Blencowe, B.J. et al. (1994) J. Cell Biol. 127, 593-607

60 Mortillaro, M.J. et al. (1996) Proc. Natl. Acad. Sci. U. S. A. 93, 8253-8257

61 Jackson, D.A., Iborra, F.J., Manders, E.M.M. and Cook, P.R. (1998) Mol. Cell. Biol. 9, 1523-1536

62 Hozak, P. et al. (1993) Cell 73, 361-373

63 Razin, S.V. et al. (1993) Cold Spring Harbor Symp. Quant. Biol. 58, 25-35

64 Ferguson, M. and Ward, D.C. (1992) Chromosoma 101, 557-565

65 Vourc'h, C., Taruscio, D., Boyle, A.L. and Ward, D.C (1993) Exp. Cell Res. 205, 142-151

66 LaSalle, J.M. and Lalande, M. (1996) Science 272, 725-728

67 Nagele, R., Freeman, T., McMorrow, L. and Lee, H. (1995) Science 270, 1831-1835

68 Buchenau, P., Saumweber, H. and Arndt-Jovin, D. (1997) J. Cell Biol. 137, 291-303

69 Shelby, R.D., Hahn, K.M. and Sullivan, K.F. (1996) J. Cell Biol. 135, 545-557

70 Marshall, W.F. et al. (1997) Curr. Biol. 7, 930-939

71 Robinett, C.C. et al. (1996) J. Cell Biol. 135, 1685-1700

72 Li, G., Sudlow, G. and Belmont, A.S. (1998) J. Cell Biol. 140, 975-989

73 Paillsson, S. et al. (1997) Exp. Cell Res. 231, 226-233

74 Spector, D.L., Goldman, R.D. and Leinwand, L.A. (1998) Cells, a Laboratory Manual (Vol. 2), Cold Spring Harbor Laboratory Press

Reference added in proof

75 Andrulis, E.D. et al. (1998) Nature 394, 592-595

J.M. Bridger and W.A. Bickmore are in the MRC Human Genetics Unit, Crewe Road, Edinburgh, UK EH4 2XU. 